\title{
On Model-Independent Analyses of Elastic Hadron Scattering
}

\author{
R. F. Ávila \\ Instituto de Matemática, Estatística e Computação Científica \\ Universidade Estadual de Campinas, UNICAMP \\ 13083-970 Campinas, SP, Brazil \\ S.D. Campos, M.J. Menon, and J. Montanha \\ Instituto de Física Gleb Wataghin \\ Universidade Estadual de Campinas, UNICAMP \\ 13083-970 Campinas, SP, Brazil
}

Received on 6 January, 2007

\begin{abstract}
By means of an almost model-independent parametrization for the elastic hadron-hadron amplitude, as a function of the energy and the momentum transfer, we obtain good descriptions of the physical quantities that characterize elastic proton-proton and antiproton-proton scattering (total cross section, $\rho$ parameter and differential cross section). The parametrization is inferred on empirical grounds and selected according to high energy theorems and limits from axiomatic quantum field theory. Based on the predictive character of the approach we present predictions for the above physical quantities at the Brookhaven RHIC, Fermilab Tevatron and CERN LHC energies.
\end{abstract}

Keywords: Elastic scattering; Hadron-induced high-energy interactions

\section{INTRODUCTION}

In the absence of a pure QCD description of high-energy elastic hadron scattering (soft diffractive processes), empirical parametrizations for the scattering amplitude and fits to the experimental data play an important role in the extraction of model-independent information on the scattering process [1]. However, one disadvantage of this kind of analysis is its local character, namely the free parameters are inferred from fits to each energy and interaction process, leading therefore to a nonpredictive approach.

In this communication, we present a first step on the development of a model-independent and predictive analysis of the elastic scattering at high energies. Specifically, we introduce a novel analytical parametrization for the imaginary part of the amplitude, inferred on empirical grounds and based on some rigorous high-energy theorems and bounds from axiomatic quantum field theory. The real part of the amplitude is analytically evaluated by means of dispersion relations (connecting, therefore, particle-particle and particle-antiparticle scattering). Fits to the experimental data on total cross section, $\rho$ parameter and differential cross section, from proton-proton $(p p)$ and antiproton-proton $(\bar{p} p)$ scattering, above $20 \mathrm{GeV}$, leads to a predictive and almost model-independent approach, able to describe quite well the bulk of the experimental data. We also present predictions for the above quantities at the RHIC, Tevatron and LHC energies.

In Sec. 2 we present the analytical parametrization and in Sec. 3 the fit results and predictions. The conclusions and some final remarks are the contents of Sec. 4.

\section{ANALYTICAL PARAMETRIZATION}

All the empirical and formal basis of our parametrization are discussed in detail in Ref. [2]. Here we shall only display the main formulae.

The imaginary part of the amplitudes for $p p$ and $\bar{p} p$ scattering are parametrized, respectively, by

$$
\frac{\operatorname{Im} F_{p p}\left(s, q^{2}\right)}{s}=\sum_{i=1}^{n} \alpha_{i}(s) e^{-\beta_{i}(s) q^{2}}
$$

with

$$
\begin{aligned}
& \alpha_{i}(s)=A_{i}+B_{i} \ln (s)+C_{i} \ln ^{2}(s), \\
& \beta_{i}(s)=D_{i}+E_{i} \ln (s)
\end{aligned}
$$

and

$$
\frac{\operatorname{Im} F_{\bar{p} p}\left(s, q^{2}\right)}{s}=\sum_{i=1}^{n} \bar{\alpha}_{i}(s) e^{-\bar{\beta}_{i}(s) q^{2}}
$$

with

$$
\begin{aligned}
\bar{\alpha}_{i}(s) & =\bar{A}_{i}+\bar{B}_{i} \ln (s)+\bar{C}_{i} \ln ^{2}(s), \\
\bar{\beta}_{i}(s) & =\bar{D}_{i}+\bar{E}_{i} \ln (s),
\end{aligned}
$$

where $A_{i}, B_{i}, \ldots E_{i}, \bar{A}_{i}, \bar{B}_{i}, \ldots \bar{E}_{i}$ are real constants (parameters to be fitted) and $i=1,2, \ldots n$.

In order that the above parametrizations might be in agreement with the rigorous results from the axiomatic quantum field theory and the analyticity-unitarity program [3], the following constraint on the free parameters must be imposed [2]: 


$$
\sum_{i=1}^{n}\left(C_{i}-\bar{C}_{i}\right)=0
$$

The corresponding real parts of the amplitudes are evaluated by means of derivative dispersion relations, extended beyond the forward direction. At high energies these relations, expressed in terms of even $(+)$ and odd $(-)$ amplitudes, are given by [4]

$$
\begin{gathered}
\frac{\operatorname{Re} F_{+}\left(s, q^{2}\right)}{s}=\frac{K}{s}+\frac{\pi}{2} \frac{\mathrm{d}}{\mathrm{d} \ln s} \frac{\operatorname{Im} F_{+}\left(s, q^{2}\right)}{s}, \\
\frac{\operatorname{Re} F_{-}\left(s, q^{2}\right)}{s}=\frac{\pi}{2}\left(1+\frac{\mathrm{d}}{\mathrm{d} \ln s}\right) \frac{\operatorname{Im} F_{-}\left(s, q^{2}\right)}{s},
\end{gathered}
$$

where $K$ is the subtraction constant.

The connections with the hadronic amplitudes are established through the usual definitions:

$$
\begin{aligned}
& F_{p p}\left(s, q^{2}\right)=F_{+}\left(s, q^{2}\right)+F_{-}\left(s, q^{2}\right) \\
& F_{\bar{p} p}\left(s, q^{2}\right)=F_{+}\left(s, q^{2}\right)-F_{-}\left(s, q^{2}\right)
\end{aligned}
$$

From parametrizations (1-4) and Eqs. (6-8) we have for the hadronic real parts:

$$
\frac{\operatorname{Re} F_{p p}\left(s, q^{2}\right)}{s}=\frac{K}{s}+\sum_{i=1}^{n}\left\{\frac{\pi}{2}\left[\alpha_{i}^{\prime}(s)-\alpha_{i}(s) \beta_{i}^{\prime}(s) q^{2}\right] e^{-\beta_{i}(s) q^{2}}+\frac{\pi}{4}\left[\alpha_{i}(s) e^{-\beta_{i}(s) q^{2}}-\bar{\alpha}_{i}(s) e^{-\overline{\beta_{i}}(s) q^{2}}\right]\right\}
$$

$$
\frac{\operatorname{Re} F_{\bar{p} p}\left(s, q^{2}\right)}{s}=\frac{K}{s}+\sum_{i=1}^{n}\left\{\frac{\pi}{2}\left[\bar{\alpha}_{i}^{\prime}(s)-\bar{\alpha}_{i}(s) \bar{\beta}_{i}^{\prime}(s) q^{2}\right] e^{-\bar{\beta}_{i}(s) q^{2}}-\frac{\pi}{4}\left[\alpha_{i}(s) e^{-\beta_{i}(s) q^{2}}-\bar{\alpha}_{i}(s) e^{-\bar{\beta}_{i}(s) q^{2}}\right]\right\},
$$

where the primes denote differentiation with respect to $\ln s$.

With this formalism we obtain analytical expressions for the main physical quantities that characterize the elastic scattering at high energies, namely the differential cross section

$$
\frac{d \sigma}{d q^{2}}=\frac{1}{16 \pi s^{2}}\left|\operatorname{Re} F\left(s, q^{2}\right)+i \operatorname{Im} F\left(s, q^{2}\right)\right|^{2},
$$

the total cross section (optical theorem)

$$
\sigma_{t o t}(s)=\frac{\operatorname{Im} F\left(s, q^{2}=0\right)}{s},
$$

and the $\rho$ parameter

$$
\rho(s)=\frac{\operatorname{Re} F\left(s, q^{2}=0\right)}{\operatorname{Im} F\left(s, q^{2}=0\right)} .
$$

\section{FITTING AND RESULTS}

We shall consider here all the experimental data available above $\sqrt{s}=20 \mathrm{GeV}$ from $p p$ and $\bar{p} p$ scattering. For the forward data on $\sigma_{t o t}$ and $\rho$, we use the Particle Data Group archives [5]. The differential cross section data concern the optical point and the data above the Coulomb-nuclear interference region. The ensemble includes 12 data sets in the region $0.01<q^{2}<14 \mathrm{GeV}^{2}$ : $p p$ scattering at $\sqrt{s}=23.5,27.4$, $30.7,44.7,52.8$, and $62.5 \mathrm{GeV}$ and $\bar{p} p$ scattering at $\sqrt{s}=31$, $53,61,546,630$ and $1800 \mathrm{GeV}$ (complete list of references to this ensemble can be found in [1].)

We performed simultaneous fits to $\sigma_{\text {tot }}(s), \rho(s)$ and $d \sigma\left(s, q^{2}\right) / d q^{2}$ data, from $p p$ and $\bar{p} p$ scattering, through the CERN-Minuit code. For this ensemble we obtained good statistical results with 3 exponential factors in Eqs. (1) and (3) and the best fit indicated $\chi^{2} / \mathrm{DOF}=2.83$ for 1277 degrees of freedom. The value of the free parameters are displayed in Table I and the results of the fit together with the experimental data are shown in Figs. 1 and 2. We see that the description of all the data is quite good.

Since we have a predictive approach, we consider extrapolations for some experiments of present interest, namely

(1) $p p$ scattering at $\sqrt{s}=200 \mathrm{GeV}$, that was investigated and might yet be investigated by the pp2pp Collaboration at the Brookhaven RHIC;

(2) $\bar{p} p$ scattering at $\sqrt{s}=1.96 \mathrm{TeV}$, that are being analyzed by the DZero Collaboration at the Fermilab Tevatron (RUN 
TABLE I: Results of the simultaneous fits to $\sigma_{t o t}, \rho$ and $d \sigma / d q^{2}$ data from $p p$ and $\bar{p} p$ scattering. All the parameters are in $\mathrm{GeV}^{-2}, K=$ $-0.1053 \pm 0.0048$ and $C_{1}=\bar{C}_{1}+\bar{C}_{2}+\bar{C}_{3}-C_{2}-C_{3}$.

\begin{tabular}{cccc}
\hline & $p p$ scattering & & $\bar{p} p$ scattering \\
\hline & & & \\
\hline$A_{1}$ & $109.70 \pm 0.28$ & $\bar{A}_{1}$ & $112.28 \pm 0.44$ \\
$B_{1}$ & $-16.529 \pm 0.039$ & $\bar{B}_{1}$ & $-0.468 \pm 0.074$ \\
$C_{1}$ & constrained & $\bar{C}_{1}$ & $-0.1673 \pm 0.0039$ \\
$D_{1}$ & $-8.91 \pm 0.32$ & $\bar{D}_{1}$ & $3.170 \pm 0.069$ \\
$E_{1}$ & $3.045 \pm 0.050$ & $\bar{E}_{1}$ & $0.4860 \pm 0.0082$ \\
$A_{2}$ & $-4.06 \pm 0.23$ & $\bar{A}_{2}$ & $10.23 \pm 0.15$ \\
$B_{2}$ & $11.387 \pm 0.030$ & $\bar{B}_{2}$ & $-6.756 \pm 0.027$ \\
$C_{2}$ & $0.0952 \pm 0.0047$ & $\bar{C}_{2}$ & $0.9613 \pm 0.0029$ \\
$D_{2}$ & $1.290 \pm 0.014$ & $\bar{D}_{2}$ & $-1.476 \pm 0.013$ \\
$E_{2}$ & $0.5097 \pm 0.0023$ & $\bar{E}_{2}$ & $0.5645 \pm 0.0012$ \\
$A_{3}$ & $1.0554 \pm 0.0077$ & $\bar{A}_{3}$ & $-8.148 \pm 0.031$ \\
$B_{3}$ & $-0.3607 \pm 0.0013$ & $\bar{B}_{3}$ & $1.2313 \pm 0.0040$ \\
$C_{3}$ & $0.02372 \pm 0.00012$ & $\bar{C}_{3}$ & $-0.05572 \pm 0.00025$ \\
$D_{3}$ & $0.6454 \pm 0.0081$ & $\bar{D}_{3}$ & $0.9272 \pm 0.0072$ \\
$E_{3}$ & $0.0176 \pm 0.0011$ & $\bar{E}_{3}$ & $0.03859 \pm 0.0011$ \\
\hline
\end{tabular}
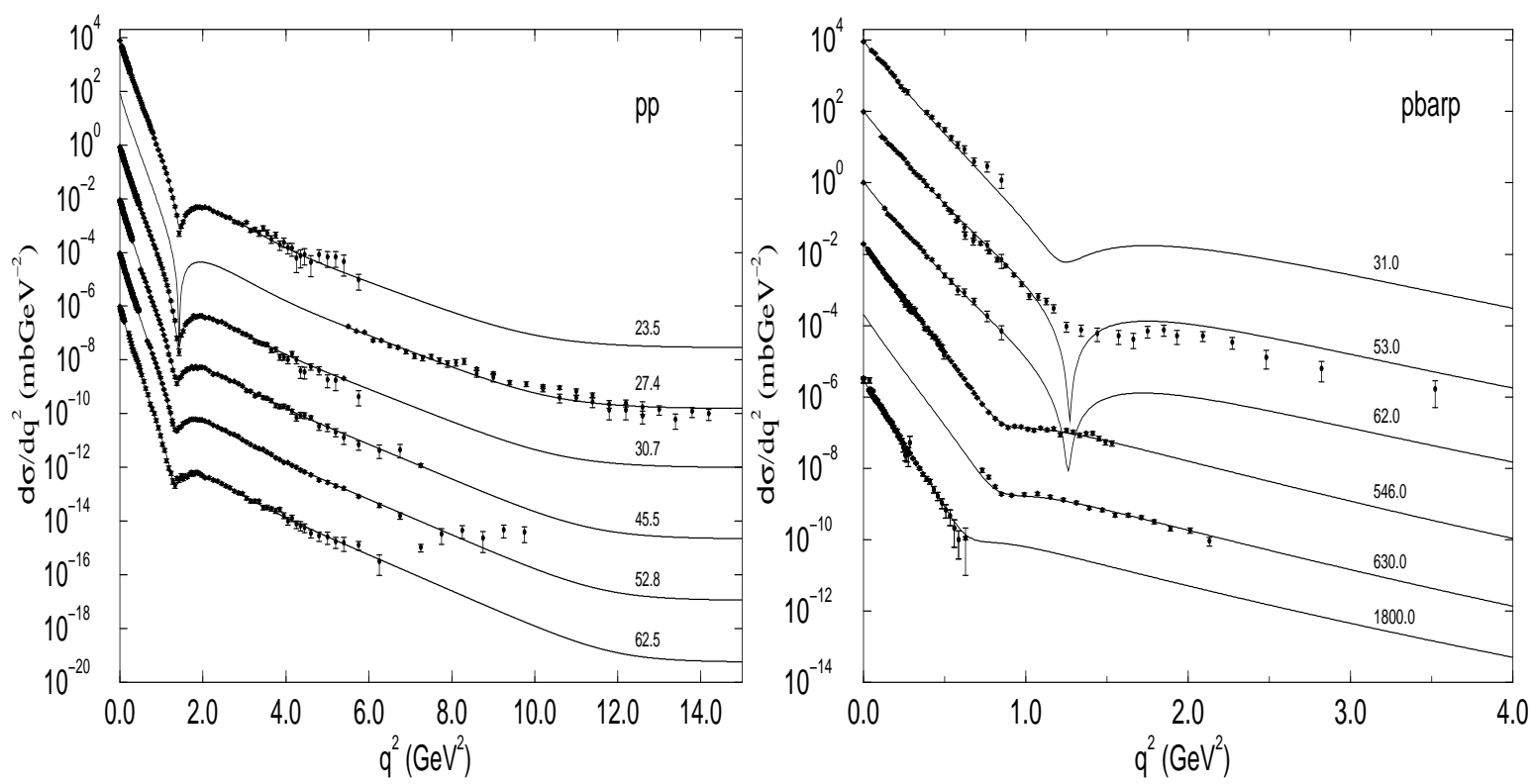

FIG. 1: The differential cross sections from global fits to $p p$ and $\bar{p} p$ data. Curves and data were multiplied by factors of $10^{ \pm 2}$.

II);

(3) $p p$ scattering at $\sqrt{s}=14 \mathrm{TeV}$, planned to be investigated by the TOTEM Collaboration at the CERN LHC.

The numerical results for the forward quantities are shown in Table II and the predicted behavior for the differential cross sections in Fig. 3.

\section{CONCLUSIONS AND FINAL REMARKS}

We have introduced an analytical parametrization for the elastic hadron-hadron scattering amplitude and a fit procedure characterized by at least four important novel aspects:
1. The parametrization is almost model-independent, with enclosed dependences on the energy and momentum extracted from the empirical behavior of the experimental data and in agreement with some high-energy theorems and bounds from axiomatic quantum field theory;

2. The real and imaginary parts of the amplitude are entire functions of the logarithm of the energy $s$ and are connected through derivative dispersion relations; the $p p$ and $\bar{p} p$ scattering are also connected to the extent that analyticity and unitarity lead to dispersion relations;

3. The approach is predictive in both energy and momentum variables; 

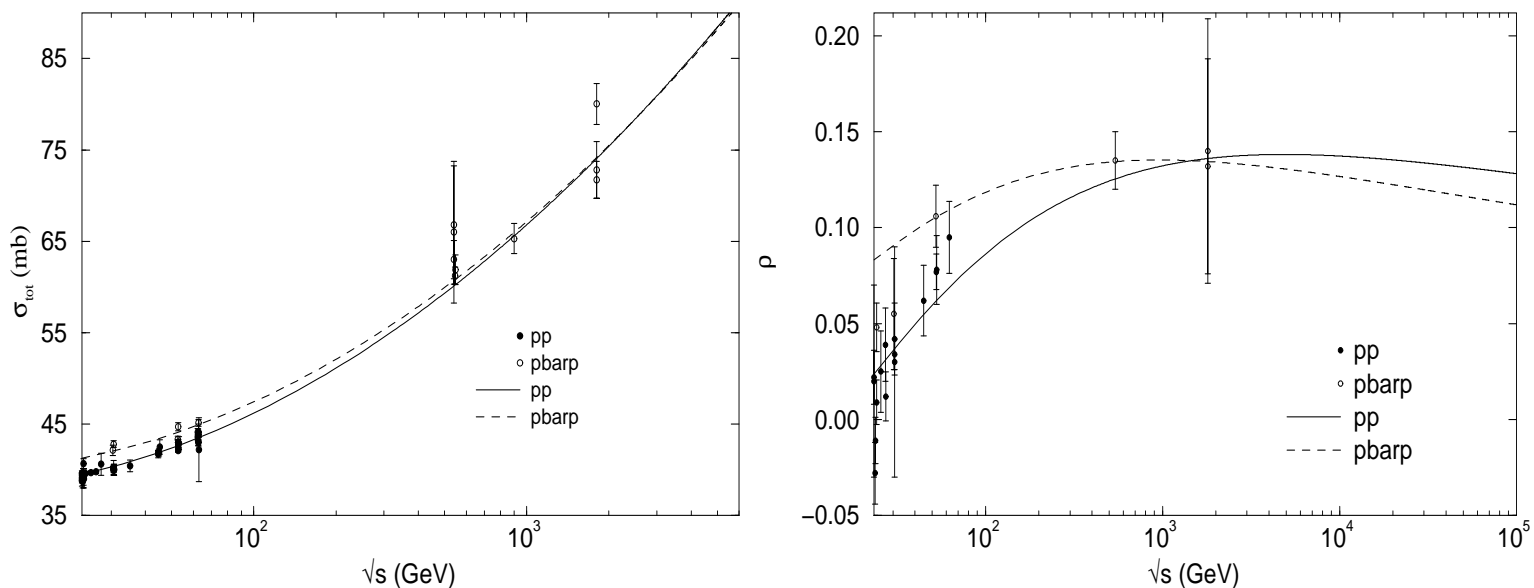

FIG. 2: Total cross sections and the $\rho$ parameter from global fits to $p p$ and $\bar{p} p$ data.

TABLE II: Predictions for $\sigma_{t o t}(s)$ and $\rho(s)$ for the quoted experiments.

\begin{tabular}{ccc}
\hline Process & $\sigma_{\text {tot }}(\mathrm{mb})$ & $\rho$ \\
\hline$p p-\sqrt{s}=14.0 \mathrm{TeV}$ & 105.4 & 0.1365 \\
$\bar{p} p-\sqrt{s}=1.96 \mathrm{TeV}$ & 75.24 & 0.1343 \\
$p p-\sqrt{s}=200 \mathrm{GeV}$ & 51.12 & 0.1065 \\
\hline
\end{tabular}

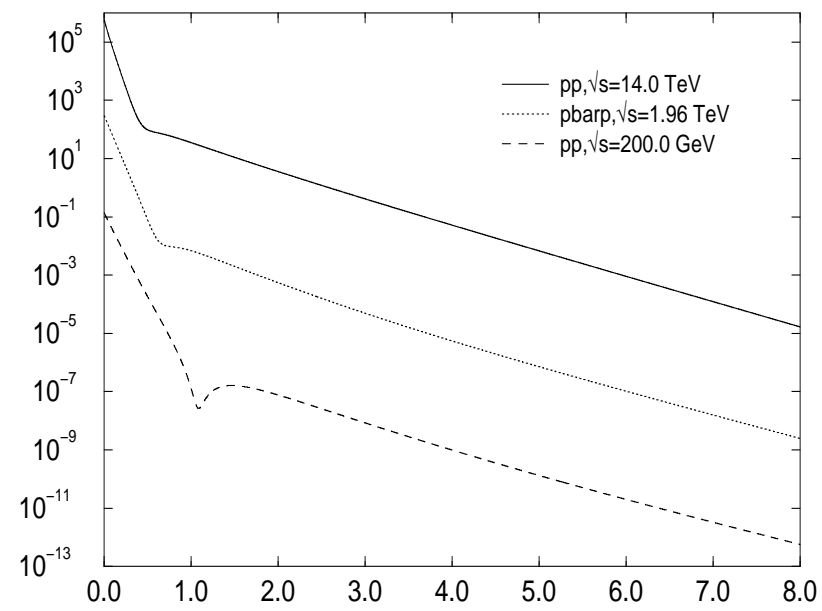

FIG. 3: Predictions for the differential cross sections at the RHIC (dashed), Tevatron (dotted) and LHC (solid) energies. The upper and lower curves were multiplied by $10^{3}$ and $10^{-3}$, respectively.
4. The fits to $p p$ and $\bar{p} p$ experimental data, above $\sqrt{s}=20$ $\mathrm{GeV}$ allow good descriptions of all the data, even in different regions of the momentum transfer (Fig. 1 ).

The results for the forward quantities indicate a crossing as the energy increases (Fig. 2), with the $p p$ total cross section and the $\rho$ parameter becoming higher than the corresponding values from $\bar{p} p$ scattering. Predictions for the quoted experiments (Fig. 3), indicate a dip for $p p$ scattering at 200 $\mathrm{GeV}$ (RHIC), located at $q^{2} \approx 1.1 \mathrm{GeV}^{2}$, and as the energy increases, the diffractive pattern becomes a shoulder for $\bar{p} p$ scattering at $1.96 \mathrm{TeV}$ (Tevatron) and $p p$ scattering at $14 \mathrm{TeV}$ (LHC). No secondary structures is predicted in the region of large momentum transfer, up to $8 \mathrm{GeV}^{2}$.

Acknowledgments. S.D.C. and M.J.M are grateful to the Organizing Committee, for the nice hospitality in Porto Alegre. For financial supports, S.D.C is thankful to CPG-IFGW and BIG-UNICAMP, R.F.A. and M.J.M. to FAPESP (Contracts No.03/00228-0 and No.04/10619-9).
[1] P.A.S. Carvalho, A.F. Martini, M.J. Menon, Eur. Phys. J. C 39, 359 (2005).

[2] R.F. Ávila, S.D. Campos, M.J. Menon, J. Montanha, hepph/0603035, to appear in Eur. Phys. J. C (2006).

[3] M. Froissart, Phys. Rev. 123, 1053 (1961); A. Martin, Il Nuovo Cimento A 42, 930 (1966); R.J. Eden, Rev. Mod. Phys. 43, 15 (1971); G. Grunberg, T.N. Truong, Phys. Rev. Lett. B 31, 63 (1973); A. Martin and F. Cheung, Analyticity Properties and Bounds on Scattering Amplitudes (Gordon and Breach Science
Publishers, New York, 1970).

[4] R.F. Ávila and M.J. Menon, Nucl. Phys. A 744, 249 (2004); J. Fischer, P. Kolár, J. Math. Phys. 25, 2538 (1984); Phys. Lett. B 64, 45 (1976).

[5] S. Eidelman et al., Phys. Lett. B 592, 1 (2004) and 2005 partial update for the 2006 edition available on the PDG www pages (URL http://pdg.lbl.gov). 